\title{
COLOR MODIFYING EFFECT OF RESIN INFILTRANT (ICON) VERSUS ADHESIVE RESIN ON AESTHETIC CORRECTION OF ENAMEL WHITE SPOT LESION: RCT
}

\author{
Rania Mosallam*, Hossam El Mandouh ** and Osama Mosallam***
}

\begin{abstract}
Objectives: Resin infiltration is an innovative approach for enhancing the esthetic appearance of white spot lesions (WSLs). The aim of this study was to compare the effect of both resin infiltrant (ICON) and adhesive resin with respect to esthetic improvement.

Methods: A randomized controlled split- mouth clinical trial was conducted on 10 young adults with at least two non-cavitated white spot lesions in esthetic zones. They were randomly assigned to two treatment groups: resin infiltrant (Icon, pre-product: DMG) or Adper ${ }^{\mathrm{TM}}$ single bond 2 adhesive system (3M ESPG dental product, USA) application after 15\% hydrochloric acid (HCL) etching and dehydration with ethanol. Vita Easyshade V (Vita Zahnfabrik, Bad Sackingen, Germany) spectrophotometer was used to measure the CIELAB coordinates and values were used to calculate difference in color $(\Delta E)$. Data were tabulated and statistically analyzed using computer software SPSS.
\end{abstract}

Results: There was a statistically significant decrease in both $(\Delta \mathrm{L})$ and $(\Delta \mathrm{a})$ with increase in $(\Delta \mathrm{b})$ with ICON application $(\mathrm{p}<0.05)$. Regarding adhesive resin, there was a significant decrease in both $(\Delta \mathrm{L})$ and $(\Delta \mathrm{a})$ coordinates with non-significant change in $(\Delta \mathrm{b})$. Also, there was a statistically significant difference between ICON and adhesive resin regarding $(\Delta \mathrm{E})$.

Conclusions: Color change of white spot lesion treated with ICON and adhesive resin was clinically obvious after treatment with hydrochloric acid. However, ICON reported significant results in esthetic improvement in all color parameters.

KEYWORDS: White spot lesions, Resin infiltration, Resin infiltrant, Adhesive resin, Esthetic.

\footnotetext{
* Associate Professor of Conservative Dentistry Department, Faculty of Oral and Dental Medicine, Cairo University ** Associate Professor, Conservative Dentistry Department, Faculty of Dentistry, Suez Canal University

*** Researcher in Restorative Dentistry and Dental Material Research Department, National Research Centre, Giza, Egypt
} 


\section{INTRODUCTION}

White enamel discolorations are defined as the enamel lesions accompanied with a reduction of the mineral content and consequently render it to opaque and chalky white appearance ${ }^{[1]}$. They can occur as a consequence of either pre- or post- eruptive damage. The pre-eruptive discoloration can arise as result of developmental disturbance such as dental fluorosis, traumatic hypo-calcification and molar-incisive hypo-mineralization. However, the post-eruptive discoloration may result from incipient caries lesion. Also, it has been reported that there is significant increase of white spot lesions after orthodontic treatments. Several techniques have been advised for treatment of esthetically objectionable defects such as improvement of remineralization, microabrasion, argon-laser irradiation, bleaching and recently resin infiltration technique. Several studies investigated the effect of resin infiltration in arresting the progression of initial caries lesions in addition to its positive effect in improving the esthetic of white spot lesions ${ }^{[8,11,18]}$.

Resin infiltration is a micro-invasive treatment option that bridge the gap between non-operative and operative interventions for masking white enamel discolorations. White spot infiltration process refers to a low-molecular weight monomer which have the capacity of flowing and filling the micropores of white spot lesions ${ }^{[4]}$. Low viscosity resin infiltrant, that have been optimized for rapid penetration into the inter-crystalline spaces, soak up the porous lesion body and sealed it. Paris et al., in 2007, developed a modifying form of the adhesive, marked as ICON, containing Bis-GAMA, TEGDMA, HEMA and ethanol in order to improve the penetration coefficient ${ }^{[15]}$. ICON are light curable resins with a very low contact angle, low viscosity and high surface tension. These properties are essential for complete penetration of the resin infiltrant into body of the lesion ${ }^{[8,12,14-17]}$.
Application of hydrochloric acid as an etchant has been estimated to be superior to $37 \%$ orthophosphoric acid gel in removing the outer surface layer of enamel lesion $(30-40 \mathrm{~mm})$ which hampers resin penetration into the lesion ${ }^{[7,10,21]}$. Studies reported that etching with orth-phosphoric acid affected the outermost $25 \mathrm{~mm}$ of the surface layer ${ }^{[1]}$. Zamorano et al. (2017), compared the penetration depth between an infiltrant resin and two adhesive systems but with use of their corresponding etching systems ${ }^{[22]}$. However, no studies were conducted to compare the esthetic changes of adhesive resin after pretreatment with $15 \% \mathrm{HCL}$ acid as an etching protocol. So, the aim of this randomized controlled study was to compare the clinical efficacy of resin infiltrant (ICON) with adhesive resin for masking white spot lesions using the same pretreatment protocol.

\section{MATERIALS AND METHODS}

\section{Ethical Considerations}

The study approval was given by ethics committee of the National Research Centre, Egypt (registration number 18033). Prior to the participation in the study, all participants signed a written informed consent.

\section{Study Design}

This study was designed as a split-mouth randomized clinical controlled trial, triple-blind (clinical evaluator, operators and patients). Enamel white spot lesions were randomly assigned into two different treatment groups: application of ICON or adhesive resin both after 15\%HCL etchant and dehydration with ethanol.

\section{Screening and Baseline Assessment}

We nudged potential participants by informing students and patients at the Ahram Canadian University Dental Clinic about the study. Inclusion criteria were: the presence of two or more 
non-cavitated white spot enamel lesions; age from 18-25; patients with good general health, adequate oral hygiene, good periodontal health, absence of caries and the giving of informed consent. Exclusion criteria were: current participant in another study, pregnancy, smoking habits, under active orthodontic treatment and incapable of contracting. At the screening appointment, eligibility of interested participant was evaluated. From the 30 screened individuals, 10 patients met the inclusion criteria, gave their informed consent, and were enrolled in the study after explaining the research protocol.

\section{Study Groups}

Ten volunteers, with at least two enamel white spot lesions that fulfilled the eligibility criteria, were selected. The treatment was conducted regarding the dental group (incisors, canines and premolars). They were divided into two groups: right side group included the right central incisor, lateral incisor and canine) and left side group included the left central incisor, lateral incisor and canine. The first restored tooth was reported for group (1) treatment, while the next tooth was automatically assigned to other treatment group (2) according to split mouth design. The isolation technique included the use of disposable saliva ejector and rubber dam system.

\section{Clinical Procedures}

Treatment was carried out by a single trained investigator at the Ahram Canadian University Dental Clinic. Enamel infiltration was applied in an accordance with the manufacturer's instructions. A rubber dam was applied to achieve clean, dry working field and protect soft tissues. The surface layer was eroded by $15 \%$ hydrochloric acid gel (Icon- Etch, supplied with the ICON kit and composed of $15 \%$ HCL, water, silica and additives; pre-product: DMG, Hamburg, Germany). It was applied with special applicator tip for 120 seconds. Care should be taken to avoid contact with adjacent tooth. To achieve homogenous etching pattern, the gel was stirred with a micro-brush from time to time during application, using the smooth surface -tips included in the product kit. Thereafter, the gel was thoroughly washed away with air water spray for 30 sec. The lesion was desiccated with ethanol (ICON Dry, supplied with kit and composed of ethanol) for 10 seconds to remove the water retained within the micro-porosities. A frosted chalky white appearance was observed on air drying. The steps of application of etchant and drying was repeated if the surface did not have a chalky white appearance.

\section{Resin Infiltrant Application}

Resin infiltrant (pre-product material supplied with ICON kit and composed of tetraethlene glycol dimethacrylte, additives, and initiators) was applied and left for 3 minutes penetration time, then excess material was removed by air blowing and flossing. The resin surplus on the surface was wiped away with a cotton roll so that the infiltrant will create a diffusion barrier inside the lesion and not on top of the lesion surface. The resin was light cured for 1 minute from the labial aspect with visible light curing unit LED (Blue phase C5) operating at light intensity of $500 \mathrm{~mW} / \mathrm{cm}^{2}$ used in standard mode. A second layer of resin infiltrant was applied and set for an additional minute and light cured for 40 seconds.

\section{Single Bond 2 Application}

Adper $^{\mathrm{TM}}$ single bond 2adhesive system (3M ESPG dental product, USA), consists of HEMA, ethanol, water, Bis-GMA, functional copolymer of polyacrylic and polyitaconic acids) was applied according to manufacturer instructions. Two coats of adhesive system were applied with a fully saturated micro-brush for $15 \mathrm{sec}$ with gentle agitation, then gently air dried for 5 seconds to evaporate solvents and light cured for 20 seconds using visible light curing unit LED (Blue phase C5). 


\section{Color Assessment}

Pretreatment and posttreatment color evaluation was done using spectrophotometer (VITA Easy shade, VITAZahnfabrik, Bad Säckingen, Germany). The VITA Easyshade is a type of spectrophotometer that function by the quantitative measurement of the reflection or transmission properties as a function of wavelength. The changes in $\mathrm{L}^{*} \mathrm{a} * \mathrm{~b} *$ values were recorded and difference in color perception $(\Delta E)$ based on CIELAB coordinates values was assessed.

\section{Statistical Analysis}

Descriptive statistics including the mean, standard deviation was calculated for each color coordinate and $\Delta \mathrm{E}$ was calculated for both groups before and after treatments. The distributions were assessed and found to be normal (Shapiro-Wilk Test). Parametric pared sample t test was used to compare the effect of each infiltrantes (intragroup analysis), while independent $t$ test was used to compare the effect of the two treatments (icon and adhesive) on the color change (intergroup analysis). The significance level used was $(\mathbf{p}=0.05)$. All statistical tests were performed using computer software SPSS (Statistics 21.0 for Windows, IBM SPSS, Armonk, NY, USA).

\section{RESULTS}

Analysis of variance demonstrated significant differences after ICON application on different color parameters Table (1), Figure (1). Statistical data interpretation concerning each parameter showed a highly statistically significant decrease in both luminance reflection $(\Delta \mathrm{L})$ and red-green coordinate $(\Delta \mathrm{a})(\mathrm{P} \leq 0.0001)$. Moreover, there was also a statistically significant increase in yellowblue coordinate $(\Delta \mathrm{b})$. Regarding adhesive resin, there was a significant decrease in both $(\Delta \mathrm{L})$ and $(\Delta \mathrm{a})$ coordinates with non-significant change in $(\Delta b)$ Table (2), Figure (2). Statistical data interpretation concerning $(\Delta \mathrm{E})$, revealed that there was a statistically significant difference between ICON and adhesive resin Table (3), Figure (3).

TABLE (1): Effect of resin infiltration(icon) on color change (CIE Lab)

\begin{tabular}{|c|c|c|c|c|c|c|}
\hline \multicolumn{4}{|c}{ Before } & \multicolumn{2}{c|}{ After } & \multicolumn{2}{c|}{ T value } & \multicolumn{2}{c|}{ P value } \\
\cline { 2 - 7 } & Mean & SD & Mean & SD & \multicolumn{1}{c|}{} \\
\hline L value & 75.5 & 5.8 & 73.3 & 5.9 & 6.26 & $.000^{* * *}$ \\
\hline a value & 2.3 & 0.5 & 1.6 & 0.4 & 5.29 & $.001^{* * *}$ \\
\hline b value & 23.6 & 4 & 27.8 & 3.9 & -3.62 & $.006^{* *}$ \\
\hline
\end{tabular}

SD: Standard deviation, *** significant,*** highly significant

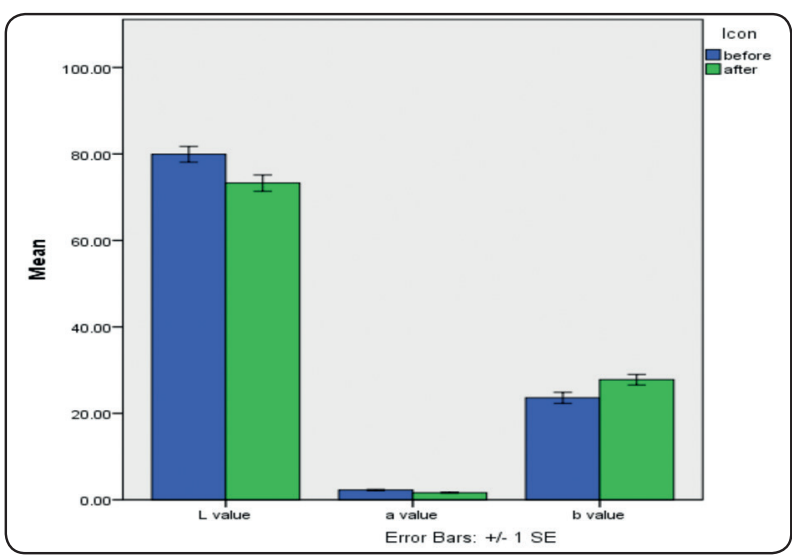

Fig. (1): Bar chart representing the mean values of $(\Delta \mathrm{L}, \Delta \mathrm{a}$, $\Delta$ b) before and after application of ICON.

TABLE (2): Effect of adhesive resin on color change (CIE Lab)

\begin{tabular}{|c|c|c|c|c|c|c|}
\hline \multirow{2}{*}{} & \multicolumn{2}{|c|}{ Before } & \multicolumn{2}{c|}{ After } & \multirow{2}{*}{ T value } & \multirow{2}{*}{ P value } \\
\cline { 2 - 6 } & Mean & SD & Mean & SD & & \\
\hline L value & 75.5 & 6.4 & 71.8 & 5.8 & 4.9 & $.001 * * *$ \\
\hline a value & 2.3 & 0.7 & 1.7 & 0.5 & 3.9 & $.003 * *$ \\
\hline b value & 27.1 & 6.4 & 28 & 5.3 & -0.79 & $.446 \mathrm{NS}$ \\
\hline
\end{tabular}

SD: Standard deviation,** significant,*** highly significant, NS: non-significant 


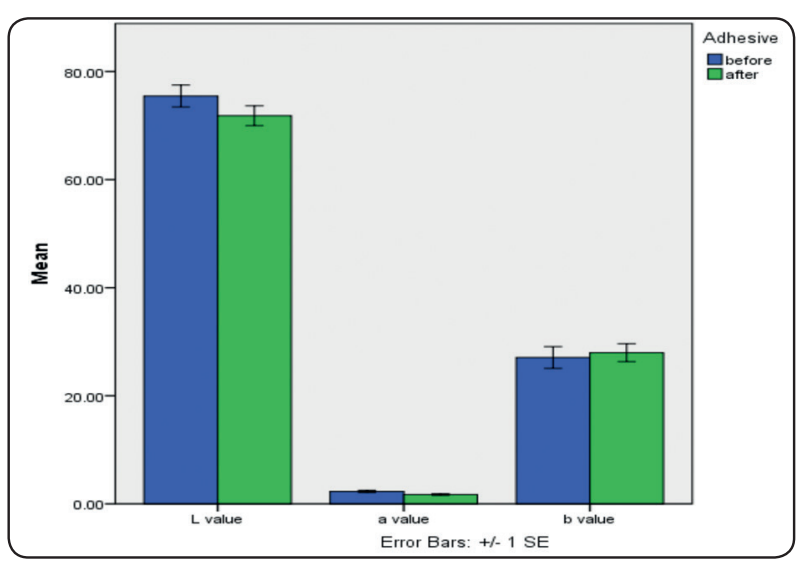

Fig. (2): Bar chart representing mean values of ( $\Delta \mathrm{L}, \Delta \mathrm{a}, \Delta \mathrm{b})$ before and after application of adhesive resin.

TABLE (3): Effect of Icon compared to adhesive using independent sample t test

\begin{tabular}{|c|c|c|c|c|c|}
\hline \multicolumn{2}{|c|}{ Icon } & \multicolumn{2}{c|}{ Adhesive } & \multirow{2}{*}{ T value } & \multirow{2}{*}{ P value } \\
\cline { 1 - 3 } Mean & SD & Mean & SD & & \\
\hline 8.8 & 3.2 & 5.1 & 2.1 & 3.048 & $.007^{* *}$ \\
\hline
\end{tabular}

SD: Standard deviation, ** significant

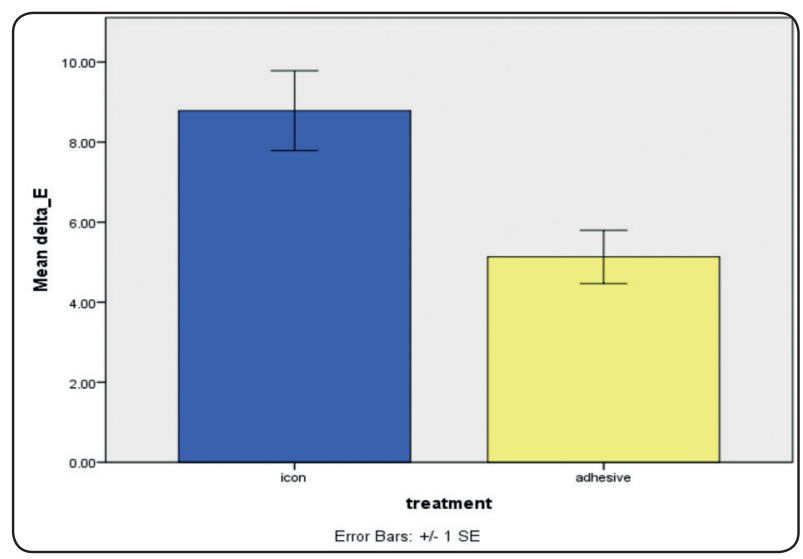

Fig. (3): Bar chart representing the mean values of $(\Delta E)$ of icon versus adhesive resin.

\section{DISCUSSION}

Resin infiltration technique is a conservative approach for esthetic correction of white spot lesions. In this randomized controlled split- mouth clinical trial we compared the performance of resin infiltrant (ICON) and adhesive resin in the same mouth.

Hydrochloric acid as pre-treatment for both resin infiltrant and adhesive resin groups was used in order to reach effective monomer penetration depth. The penetration depth of $15 \%$ HCL etching is more than twice that obtained with phosphoric acid thus enabling deeper penetration into the lesion ${ }^{[7,10]}$. Paris et al. (2007), reported penetration differences between $15 \% \mathrm{HCl}$ gel and $37 \%$ orthophosphoric acid gel of $58 \mathrm{~mm}$ versus $18 \mathrm{~mm}$ respectively ${ }^{[15]}$. The rational of using ethanol wet bonding technique is to improve the efficacy of penetration of infiltrant as it will coat hydrophobic monomers to infiltrate into wet enamel or dentin ${ }^{[5,9]}$. The color of desiccated surface lesion should change during ethanol application otherwise the ethanol will not reach the body of the lesion which could justify the outcome.

Owing to the subjective and inconsistent results of visual shade assessment, VITA Easyshade color measuring device was used. The pretreatment and post-treatment CIE $\mathrm{L}^{*} \mathrm{a} * \mathrm{~b} *$ values were recorded using Vita Easyshade and $\Delta \mathrm{E}$ was calculated. Although $\Delta \mathrm{E}$ itself does not absolutely assess the color change since the values is always positive, it is of importance to assess the absolute color parameters L*a*b* values. So, quantitative comparison was done by evaluation of the absolute color parameters $\mathrm{L}, \mathrm{a}^{*}$, and $\mathrm{b}^{*}$.

When analyzing the color parameters, the lightness $\left(\mathrm{L}^{*}\right)$ decreased in both groups with highly statistically significant difference before and after treatment. This reduction could be explained by spectral components with decrease in reflectance $\%$ at all waves lengths. Moreover, the red green color balance $\left(\mathrm{a}^{*}\right)$ was shifted toward green color in both treatments but with highly statistically significant difference in resin infiltrant group. Lower $a^{*}$ values 
could be attributed to the lower reflectance of light that locates in the green zone of color spectral. Although there was no statistically significant difference in $\Delta \mathrm{b}$ value among adhesive resin group but there was a statistically significant difference in resin infiltrant group with an increase in $b^{*}$ value (yellow directions) was detected.

Moreover, using the criterion for perceptible color change of $(\Delta E \geq 3.5)$, showed that, obvious improvement in color could be found with both ICON and adhesive resin but with statistically significant change with ICON. This result is in accordance with previous results indicating an improvement of esthetic appearance of resin infiltrated white spot lesions ${ }^{[2,17]}$. The principle of masking of white spot lesion by resin infiltrant is based on changed in optical properties as result of light scattering. In white spot lesion, the micropores of enamel were filled with water or air with refractive index of 1.33 and 1.0 respectively which is lower in value than the refractive index of sound enamel $(\mathrm{RI}=1.62)$. However, the refractive index of resin infiltrant is 1.46 . Therefore, when these lesions are filled with resin infiltrant, the difference in refractive indices between sound tooth and resin filled porosities will be negligible and lesions blends with the surrounding enamel. In addition, it requires no shade selection due to its chameleon effect. Moreover, the penetration depth of resin infiltrant is superior than adhesive resin due to its higher penetration coefficient (PC). Van Landuyt et al. 2007, stated that the high content of TEGDMA that have a low viscosity, low molecular weight and with high conversion degree confer a great potential for penetration ability ${ }^{[20]}$. Mayer-Lueckel and Paris ,in 2008, reported that the penetration depths of the adhesive were significantly lower in comparison to the resin infiltrant as result of difference in penetration coefficients between the adhesive and infiltrating resins with $\mathrm{PC}=31 \mathrm{~cm} / \mathrm{sec}$ and $273 \mathrm{~cm} /$ sec respectively ${ }^{[12]}$. Munoz et al. 2013, concluded that resin infiltration technique showed visually perceptible improvements for mild to moderate fluorosis and hypoplasia stains ${ }^{[14]}$.
In addition, the perceptible color change with adhesive resin could be attributed to effective monomer penetration depth obtained after 15\% HCL pre-treatment. Reviewing the results of penetration depth, we found different results. Zamorano et al. 2017, concluded that these differences could be attributed to the origin of white spot lesion whether natural or artificially induced, surface pretreatment, infiltrate characteristics, infiltrant chemical composition, application time and the use of different microscopic observation techniques. They compared the penetration depth between an infiltrant and two adhesive systems using corresponding manufacturer protocol and found that the penetration depth of infiltrant ICON was statistically significantly higher compared to the penetration depth of XP-Bond and Single Bond 2 adhesive systems. They attributed that to the high molecular weight and a highly viscous monomer (Bis-GMA) and HEMA, that controls its penetration ${ }^{[22]}$. In addition, colloidal silica was added as a filler to modify resin viscosity thus explaining the lower values reached for resin tag length ${ }^{[20]}$.

Under the tested conditions, ICON resin infiltrant obtained significant improvement in color of white spot lesion than adhesive resin. However, there are other factors should be kept in consideration that also affect the penetration efficacy of resin materials such as sealing quality, surface microhardness, resistance to acid attack. So, long term studies are essential to report the effectiveness and stability of these materials.

\section{CONCLUSIONS}

Within the limitation of the study, it can be concluded that color change of white spot lesion treated with ICON and adhesive resin was clinically obvious after treatment with hydrochloric acid. However, the outcome results with ICON was much more better than adhesive resin. Further long-term studies are essential for evaluation of the stability of esthetic improvement. 


\section{ACKNOWLEDGMENT}

The authors would like to thank Abd-Elmageed Alam eldeen (Fifth year student, Ahram Canadian University, Giza, Egypt) for his participation in the clinical work for partial fulfillment of the graduation requirements.

\section{DISCLOSURE}

The authors do not have any financial interest regarding the products discussed in this study.

\section{REFERENCES}

1. Basaran G, Veli I, Basaran E. Non-cavitated approach for the treatment of white spot lesions: A case report. International Dental Research. 2011;1:65-9.

2. Borges A, Caneppele T, Masterson D, Maia L. Is resin infiltration an effective esthetic treatment for enamel developmental defects and white spot lesions? A systematic review. J. of Dentistry.2017; 56: 11-18.

3. Crombie F, Manton D, Palamar J, Reynolds E. Resin infiltration of developmentally hypomineralized enamel. Int.Pediatr. 2014; 24:51-55.

4. Davila J, Buonocuore M, Greeley C, Provenza D. Adhesive penetration in human artificial and natural white spots. Journal of Dental Research. 1975;54:998-1008.

5. de Barros L, Apolonio F, Loguercio A, de Saboia V. Resindentin bonds of etch-and -rinse adhesives to alcoholsaturated acid-etched dentin. The Journal of adhesive dentistry. 2013;15: 333-40.

6. Denis M, Atlan A, Vennat E, Tirlet G, Attal J. White defects on enamel: diagnosis and anatomomapathology: two essential factors for proper treatment (part 1). Int. Orthod. 2103; 11:139-165.

7. Gugnani N, Pandit I, Gupta M, Josan R. Caries infiltration of noncavitated white spot lesions: A novel approach for immediate esthetic improvement. Contemporary Clinical Dentistry.2012; 3: 199-202.

8. Kielbassa AM, Muller J, Gernhardt CR. Closing the gap between oral hygiene and minimally invasive dentistry: a review on the resin infiltration technique of incipient (proximal) enamel lesions. Quintessence Int. 2009; 40:663-81.

9. Li F, Liu X, Zhang L, Kang J, Chen J. Ethanol-wet bonding technique may enhance the bonding performance of contemporary etch and -rinse dental adhesives. The Journal of Adhesive Dentistry. 2012; 40: 713-8.

10. Meyer-Lueckel H, Paris S, Kielbassa AM. Surface layer erosion of natural caries lesions with phosphoric and hydrochloric acid gels in preparation for resin infiltration. Caries Re . 2007; 41: 223-30.

11. Meyer-Lueckel H and Paris S. Progression of artificial enamel caries lesions after infiltration with experimental light curing resins. Caries Res. 2008a; 42:117-24.

12. Meyer-Lueckel $\mathrm{H}$ and Paris $\mathrm{S}$. Improved resin infiltration of natural caries lesions. J. Dent Res. 2008b; 87:1112-6.

13. Meyer-Lueckel H and Paris S. Masking of labial enamel white spot lesions by resin infiltration- A clinical report. Quintessence Int. 2009; 40:713-718.

14. Muñoz MA, Arana-Gordillo LA, Gomes GM, Gomes OM, Bombarda NH, Reis A, Loguercio AD. Alternative esthetic management of fluorosis and hypoplasia stains: blending effect obtained with resin infiltration techniques. J Esthet Restor Dent. 2013;25:32-9.

15. Paris S, Meyer-Lueckel H, Colfen H, Kielbassa AM . Resin infiltration of artificial enamel caries lesions with experimental light curing resins. Dent Mater J. 2007a; 26:582-588.

16. Paris $\mathrm{S}$, Meyer-Lueckel H, Kielbassa AM. Resin infiltration of natural caries lesions. J Dent Res .2007b;86:662-666.

17. Paris S, Meyer-Lueckel H . Masking of labial enamel white spot lesions by resin infiltration- a clinical report. Quintessence Int. 2009; 40: 713-718.

18. Paris S, Meyer-Lueckel H. Inhibition of caries progression by resin infiltration in situ. Caries Res. 2010; 44:47-54.

19. Paris S, Schwendicke F, Keltsch J, Dorfer C, MeyerLueckel H. Masking of white spot lesions by resin infiltration in vitro. J. Dent. 2013; 41:28-34

20. Van Landuyt K, Snauwaert J, De Munck J, Peumans M, Yoshida Y, Poitevin A, Coutinho E, Suzuki K, Lambrechts P, Van Meerbeek B. Systematic review of the chemical composition of contemporary dental adhesives. Biomaterials, 2007;28: 3757-3785.

21. Yim H-K, Min J, Kim B. Modification of surface pretreatment of white spot lesions to improve the safety and efficacy of resin infiltration. Korean J. of Orthodontics. 2014; 44:195-202.

22. Zamorano X, Valenzuela V, Daniela A, Ituraun A. SEM comparison of penetration in artificial white spot lesion between an infiltrant resin and two adhesive systems. Open J. of Stomatology. 2017; 7: 147-157. 